OPEN ACCESS

Edited by:

Jingyan Han,

Boston University, United States

Reviewed by:

Guiqian Chen,

Zhejiang Sci-Tech University, China Yajing Wang,

Thomas Jefferson University United States

*Correspondence:

Jian Hou

chnhouj@163.com

Specialty section:

This article was submitted to Cardiovascular Therapeutics,

a section of the journal

Frontiers in Cardiovascular Medicine

Received: 12 June 2021

Accepted: 29 September 2021

Published: 25 October 2021

Citation:

Hu B, Xu G, Jin X, Chen D, Qian X,

$L i W, X u L$, Zhu J, Tang J, Jin $X$ and

Hou J (2021) Novel Prognostic

Predictor for Primary Pulmonary

Hypertension: Focus on Blood Urea

Nitrogen.

Front. Cardiovasc. Med. 8:724179.

doi: $10.3389 /$ fcrm.2021.724179

\section{Novel Prognostic Predictor for Primary Pulmonary Hypertension: Focus on Blood Urea Nitrogen}

\author{
Bo Hu${ }^{1}$, Guangtao $X u^{2}$, Xin Jin ${ }^{2}$, Deqing $\mathrm{Chen}^{2}$, Xiaolan Qian ${ }^{1}$, Wanlu $\mathrm{Li}^{2}$, Long $X u^{2}$, \\ Jia Zhu ${ }^{2}$, Jie Tang ${ }^{1}$, Xiuhui Jin ${ }^{3}$ and Jian Hou ${ }^{4 *}$ \\ ${ }^{1}$ Department of Pathology and Municipal Key-Innovative Discipline of Molecular Diagnostics, Jiaxing Hospital of Traditional \\ Chinese Medicine, Jiaxing University, Jiaxing, China, ${ }^{2}$ Forensic and Pathology Laboratory, Department of Pathology, Institute \\ of Forensic Science, Jiaxing University, Jiaxing, China, ${ }^{3}$ Department of Immunology and Human Biology, University of \\ Toronto, Toronto, ON, Canada, ${ }^{4}$ Department of Cardiac Surgery, The First Affiliated Hospital of Sun Yat-sen University, \\ Guangzhou, China
}

Background: Primary pulmonary hypertension (PPH) is a life-threatening disease associated with increased mortality. The urea cycle pathway plays a major role in PPH severity and treatment response. Little is known about the association of the blood urea nitrogen (BUN) and PPH prognosis.

Methods: Clinical data were extracted from the Medical Information Mart for Intensive Care III (MIMIC-III) database. Adult patients ( $\geq 18$ years) patients with primary pulmonary hypertension $(\mathrm{PPH})$ in the database were enrolled. Spearman correlation was used to analyze the association of BUN with length of hospital and intensive care unit (ICU) stays. The chi-square test was used to analyze the association of BUN with mortality rate. Survival curves were estimated using the Kaplan-Meier method and compared by the log-rank test. Multivariable logistic regression was used to identify the BUN as an independent prognostic factor of mortality. Receiver operating characteristic (ROC) curves and the area under the curve (AUC) were used to analyze the sensitivity and specificity for mortality.

Results: In total, 263 patients who met the selection criteria were enrolled. BUN was significantly positively associated with length of hospital stay and ICU stay (hospital stay: $\rho=0.282$, ICU stay: $\rho=0.276$; all $P<0.001$ ). Higher hospital, 90-day and 4-year mortality rates were observed in the higher BUN quartile of $\mathrm{PPH}$ patients (hospital: $P$ $=0.002$; 90-day: $P=0.025$; 4-year: $P<0.001$ ). The Kaplan-Meier survival curves showed that patients in higher BUN quartile tended to have lower 4-year survival (Q1:7.65\%, Q2: 10.71\%; Q3: 14.80\%, Q4: 16.84\%; $P<0.0001$ ). Logistic regression analyses found a significant association of BUN and mortality (hospital: $\mathrm{OR}=1.05$, $95 \% \mathrm{Cl}=1.02-1.08, P=0.001 ; 90$-day: $\mathrm{OR}=1.02,95 \% \mathrm{Cl}=1.00-1.05, P=$ 0.027 ; 4-year: $\mathrm{OR}=1.05,95 \% \mathrm{Cl}=1.02-1.08, P=0.001$ ). Results of $\mathrm{ROC}$ and $\mathrm{AUC}$ showed that the diagnostic performance of BUN for mortality was moderately good. 
Conclusion: BUN was positively correlated with the length of hospital stay and ICU stay of PPH patients. Higher BUN was associated with higher hospital, 90-day and 4-year mortality and lower 4-year survival of $\mathrm{PPH}$ patients. These findings indicate that BUN can be a novel potential prognostic predictor for $\mathrm{PPH}$.

Keywords: primary pulmonary hypertension, pulmonary hypertension, blood urea nitrogen, prognostic predictor, intensive care unit, pulmonary arterial hypertension, medical information mart for intensive care III (MIMIC-III)

\section{INTRODUCTION}

Pulmonary hypertension $(\mathrm{PH})$ is a rare, progressive disease that affects the precapillary pulmonary vasculature, and its exact underlying risk factors are still unknown (1). Pulmonary artery pressure is persistently more than $25 \mathrm{mmHg}$ at rest and more than $30 \mathrm{mmHg}$ during exercise, which could ultimately lead to right ventricular (RV) failure and death $(2,3)$. PH is classified into 5 clinical subgroups in the World Health Organization's (WHO) classification system, and primary pulmonary hypertension $(\mathrm{PPH})$ belongs to group 1 , in which pulmonary arterial hypertension (PAH) is idiopathic (1).

Decreased nitric oxide (NO) is considered to be an important pathogenetic mechanism in $\mathrm{PAH}$ (4). The urea cycle provides arginine, and then arginine is transformed to citrulline, NO and water by nitric oxide synthase (NOS) $(5,6)$. In PAH patients, arginase activity is increased and then competes with NOS, resulting in decreased arginine and NO production (7-9). Arginase is the enzyme that can transform arginine to ornithine and urea, which can compete with NOS for arginine, leading to decreasing NO expression (10). An increase in urea was observed in experimental pulmonary arterial hypertension rats (11); thus, it has been proposed that increasing urea levels might be associated with $\mathrm{PH}$ progression. However, little is known about the urea amount associated with $\mathrm{PH}$ progression and prognosis.

In the present study, we aimed to investigate the association between the blood urea nitrogen (BUN) and the length of hospital stay and intensive care unit (ICU) stay, the hospital mortality, 90-day mortality and 4-year mortality of the patients with PPH.

\section{METHODS}

\section{Data Source}

A retrospective cohort study design was used in this study. Data were obtained from the ICU database, a free accessible critical care database of Medical Information Mart for Intensive Care III (MIMIC-III). The clinical data of patients who stayed in the ICU of Beth Israel Deaconess Medical Center (BIDMC) between 2001 and 2012 were selected (12). The institutional review boards of both the BIDMC and the Massachusetts Institute of Technology Affiliates approved the access to the database. No informed consent was required because all of the data were deidentified.

\section{Patient Selection}

Clinical data of eligible patients in the MIMIC-III database were selected for entry into this study. The eligibility criteria were:
(1) patients diagnosed with PPH (the PPH was identified using International Classification of Diseases, Ninth Revision, Clinical Modification (ICD-9-CM) codes. For PPH, the ICD-9-CM code was 4160 in MIMIC-III database); (2) PPH patients older than 18 years; and (3) PPH patients with routine blood examinations within $24 \mathrm{~h}$ of admission (including levels of BUN, creatinine, peripheral white blood cell count (WBC), serum sodium, glucose and platelets). The exclusion criteria of this study: (1) patients without PPH; (2) PPH patients younger than 18 years; (3) $\mathrm{PPH}$ patients with incomplete blood test indicators; and (4) $\mathrm{PPH}$ patients with diseases involved in alterations of BUN and/or creatinine levels (acute or chronic kidney disease, kidney trauma, primary or metastatic tumors of the kidney, etc.).

\section{Data Extraction}

All of the data were obtained and extracted by using the Structured Query Language (SQL), and pgAdmin4 for PostgreSQL was used as the administrative platform. The extracted data mainly included demographics (age and sex), vital signs [diastolic blood pressure (DBP), heart rate (HR), respiratory rate (RR), systolic blood pressure (SBP), percutaneous oxygen saturation $\left(\mathrm{SpO}_{2}\right)$, and temperature], comorbidities (congestive heart failure, cardiac arrhythmias, valvular disease, pulmonary circulation disorder, peripheral vascular, hypertension, chronic pulmonary, uncomplicated diabetes, complicated diabetes, liver disease and renal failure), laboratory parameters (WBC, platelet count, BUN, serum creatinine, serum glucose, serum potassium, and serum sodium), the Simplified Acute Physiology Score (SAPS) II and the Sequential Organ Failure Assessment (SOFA) score. For BUN and creatinine, the intimal value represented the initial value measurement after admission. The max value represented the maximum value measured during hospitalization, and the value min represented the minimum value measured during hospitalization. Given that the proportion of missing data for each variable was $<1.5 \%$, we directly omitted these data in further analyses.

\section{Outcome Variables}

The following outcome variables were extracted: hospital mortality, length of ICU stay, length of hospital stay, 90-day mortality (post-ICU admission) and 4-year mortality. Because a patient may have had more than one ICU admission during a single hospitalization, the length of ICU stay was entirely determined by the first ICU hospitalization. For 4-year mortality, only patients in the CareVue system who were followed for at least 4 years were analyzed. 
TABLE 1 | Baseline characteristics of the study population with different survival status in hospital.

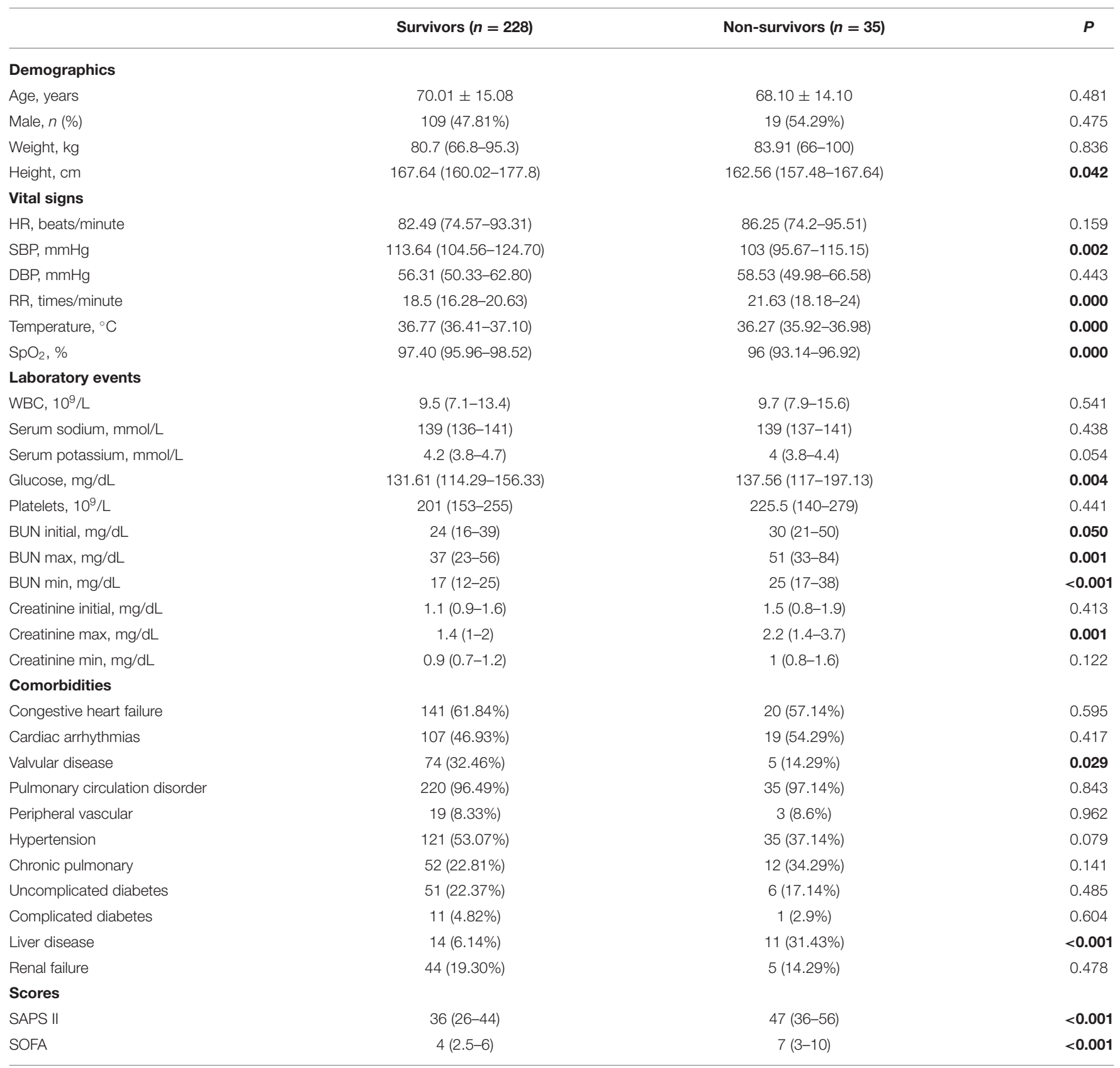

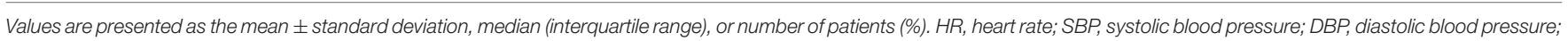

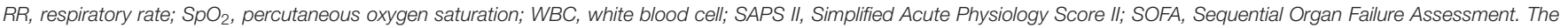
bold values represents a $P$ value $<0.05$.

\section{Statistical Analysis}

Continuous variables are presented as the mean \pm standard deviation or median (interquartile range), and were compared via $t$-test or the Mann-Whitney $U$-tests. Categorical data are presented as numbers with proportions and were analyzed via the $\chi^{2}$-test. The correlation of the length of ICU stay and hospital stay with the laboratory parameter was assessed with the nonparametric Spearman's rank correlation test. Survival curves were estimated using the Kaplan-Meier method and compared by the log-rank test. Logistic regression with the univariate/multivariate analyses was used to identify independent prognostic factors of mortality (hospital, 90-day and 4-year mortality) for PPH. Three different models were designed to adjust for potential confounders. Model 1 was adjusted for RR, $\mathrm{SpO}_{2}$, liver disease, valvular disease, SAPS II score and SBP. Model 2 was adjusted for $\mathrm{RR}, \mathrm{SpO}_{2}$ and SOFA score. Model 3 was adjusted for RR, $\mathrm{SpO}_{2}$, liver disease, valvular disease, SAPS II score, SBP and SOFA score. Receiver operating characteristic (ROC) curves and the area under the curve (AUC) were used to analyze the sensitivity and specificity. All statistical analyses were performed using STATA, 
TABLE 2 | The correlation of BUN and creatinine with hospital stay and ICU stay.

\begin{tabular}{lcccccc}
\hline & \multicolumn{2}{c}{ Length of hospital stay } & & \multicolumn{2}{c}{ Length of ICU stay } \\
\cline { 2 - 3 } & Spearman's rho & $\boldsymbol{P}$ & & Spearman's rho & $\boldsymbol{P}$ \\
\hline BUN initial, mg/dL & 0.116 & 0.061 & & 0.175 & $\mathbf{0 . 0 0 5}$ \\
BUN max, mg/dL & 0.282 & $<\mathbf{0 . 0 0 1}$ & & 0.276 & $<\mathbf{0 . 0 0 1}$ \\
BUN min, mg/dL & -0.046 & 0.454 & & 0.026 & 0.676 \\
Creatinine max, mg/dL & 0.224 & $<\mathbf{0 . 0 0 1}$ & & 0.183 & $\mathbf{0 . 0 0 3}$ \\
\hline
\end{tabular}

The bold values represents a $P$ value $<0.05$.

TABLE 3 | The relationship between BUN and creatinine with hospital mortality.

\begin{tabular}{|c|c|c|c|c|c|}
\hline & Q1 & Q2 & Q3 & Q4 & $P$ \\
\hline \multicolumn{6}{|l|}{ BUN initial } \\
\hline Survivors & 66 (97.06\%) & 53 (81.54\%) & 55 (84.62\%) & 54 (83.08\%) & 0.032 \\
\hline Non-survivors & 2 (2.94\%) & 12 (18.46\%) & 10 (15.38\%) & 11 (16.92\%) & \\
\hline \multicolumn{6}{|l|}{ BUN max } \\
\hline Survivors & 65 (92.86\%) & 60 (88.24\%) & 53 (85.48\%) & 50 (79.37\%) & 0.141 \\
\hline Non-survivors & $5(7.14 \%)$ & $8(11.76 \%)$ & $9(14.52 \%)$ & 13 (20.63\%) & \\
\hline \multicolumn{6}{|l|}{ BUN min } \\
\hline Survivors & 68 (95.77\%) & 60 (89.55\%) & 55 (85.94\%) & 45 (73.77\%) & 0.002 \\
\hline Non-survivors & $3(4.23 \%)$ & 7 (10.45\%) & $9(14.06 \%)$ & 16 (26.23\%) & \\
\hline \multicolumn{6}{|c|}{ Creatinine max } \\
\hline Survivors & 61 (91.04\%) & 64 (95.52\%) & 54 (88.52\%) & 49 (72.06\%) & $<0.001$ \\
\hline Non-survivors & 6 (8.96\%) & $3(4.48 \%)$ & 7 (11.48\%) & 19 (27.94\%) & \\
\hline
\end{tabular}

TABLE 4 | The relationship between BUN and creatinine with 90-day mortality.

\begin{tabular}{lccccc}
\hline \multicolumn{1}{c}{ Q1 } & Q2 & Q3 & Q4 & P \\
\hline BUN initial & & & & & \\
Survivors & $62(91.18 \%)$ & $51(78.46 \%)$ & $49(75.38 \%)$ & $49(75.38 \%)$ & 0.066 \\
Non-survivors & $6(8.82 \%)$ & $14(21.54 \%)$ & $16(24.62 \%)$ & $16(24.62 \%)$ & \\
BUN max & & & & & \\
Survivors & $60(85.72 \%)$ & $57(83.82 \%)$ & $48(77.42 \%)$ & $46(73.02 \%)$ & 0.235 \\
Non-survivors & $10(14.29 \%)$ & $11(16.18 \%)$ & $14(22.58 \%)$ & $17(26.98 \%)$ & \\
BUN min & & & & & \\
Survivors & $65(91.55 \%)$ & $54(80.60 \%)$ & $48(75.00 \%)$ & $44(72.13 \%)$ & $\mathbf{0 . 0 2 5}$ \\
Non-survivors & $6(8.45 \%)$ & $13(19.40 \%)$ & $16(25.00 \%)$ & $17(27.87 \%)$ & \\
Creatinine max & & & & & \\
Survivors & $54(80.60 \%)$ & $59(88.06 \%)$ & $51(83.61 \%)$ & $47(69.12 \%)$ & $\mathbf{0 . 0 4 0}$ \\
Non-survivors & $13(19.40 \%)$ & $8(11.94 \%)$ & $10(16.39 \%)$ & $21(30.88 \%)$ & \\
\hline
\end{tabular}

$Q$, Quartile of serum BUN and creatinine value. The bold values represents a $P$ value $<0.05$.

version 14.0 (StataCorp, College Station, TX). $P$-values of $<0.05$ were considered to indicate statistical significance.

\section{RESULTS}

\section{Baseline Characteristics of the Study Population}

In total, 263 patients who met the selection criteria were enrolled in our study, among whom 35 patients (13.3\%) died in the hospital. The baseline characteristics of the enrolled patients are
TABLE 5 | The relationship between BUN and creatinine with 4-year mortality.

\begin{tabular}{lccccc}
\hline \multicolumn{1}{c}{ Q1 } & Q2 & Q3 & Q4 & $\boldsymbol{P}$ \\
\hline BUN initial & & & & & \\
Survivors & $44(81.48 \%)$ & $29(58.00 \%)$ & $12(28.57 \%)$ & $13(30.95 \%)$ & $<\mathbf{0 . 0 0 1}$ \\
Non-survivors & $10(18.52 \%)$ & $21(42.00 \%)$ & $30(71.43 \%)$ & $29(69.05 \%)$ & \\
BUN max & & & & & \\
Survivors & $40(68.97 \%)$ & $31(60.78 \%)$ & $13(30.23 \%)$ & $14(31.82 \%)$ & $<\mathbf{0 . 0 0 1}$ \\
Non-survivors & $18(31.03 \%)$ & $20(39.22 \%)$ & $30(69.77 \%)$ & $30(68.18 \%)$ & \\
BUN min & & & & & \\
Survivors & $41(73.21 \%)$ & $28(57.14 \%)$ & $19(39.58 \%)$ & $10(23.25 \%)$ & $<\mathbf{0 . 0 0 1}$ \\
Non-survivors & $15(26.79 \%)$ & $21(42.86 \%)$ & $29(60.42 \%)$ & $33(76.74 \%)$ & \\
Creatinine max & & & & & \\
Survivors & $33(63.46 \%)$ & $33(64.71 \%)$ & $17(36.96 \%)$ & $15(31.91 \%)$ & $\mathbf{0 . 0 0 1}$ \\
Non-survivors & $19(36.54 \%)$ & $18(35.29 \%)$ & $29(63.04 \%)$ & $32(68.09 \%)$ & \\
\hline
\end{tabular}

For 4-year mortality, only patients in the CareVue system who were followed for at least 4-year were analyzed. Q, Quartile of serum BUN and creatinine value. The bold values represents a $P$ value $<0.05$.

briefly summarized in the Table $\mathbf{1}$, including demographics, vital signs, laboratory events, comorbidities and scores.

The demographic characteristics of the survivors and non-survivors are presented in Table 1. No significant difference was observed for age, sex or weight between non-survivors and survivors. Non-survivors had much higher BUN values (initial: 24 vs. $30, P=0.050$; $\max : 37$ vs. $51, P=0.001$; $\min : 17$ vs. $25, P<0.001$ ) and serum creatinine level (max: 1.4 vs. 2.2 , $P=0.001$ ). Non-survivors tended to have lower height, SBP, temperature and $\mathrm{SpO}_{2}$ values and higher RR, glucose, SAPS II scores, and SOFA score, as well as a history of valvular disease and liver disease.

\section{The Prognostic Significance of BUN for PPH}

To investigate the association of BUN with the length of hospital stay and ICU stay in PPH patients, Spearman's rank correlation test was used, and the results are shown in Table 2. BUN max was significantly positively associated with length of hospital stay and ICU stay (hospital stay: Spearman's rho $=0.282, P<0.001$; ICU stay: Spearman's rho $=0.276, P<0.001$ ). Initially, BUN was significantly positively associated only with length of ICU stay (Spearman's rho $=0.175, P=0.005$ ). Moreover, creatinine max was positively associated with length of hospital stay and ICU stay (hospital stay: Spearman's rho $=0.224, P<0.001$; ICU stay: Spearman's rho $=0.183, P=0.003$ ).

Furthermore, the correlation of BUN with the mortality of PPH patients was investigated. Quartiles of BUN initial and BUN min were significantly correlated with hospital mortality (BUN initial: $P=0.032$; BUN min: $P=0.002$ ) (Table 3). A lower rate of hospital mortality was observed in the patients in the first BUN initial quartile than those in the second, the third and the fourth quartiles. For BUN min, a higher rate of hospital mortality was observed in the higher quartile of PPH patients. Furthermore, a much higher rate of hospital mortality was observed in the patients in the fourth creatinine max quartile than in the patients in the other quartiles $(P<0.001)$ (Table 3$)$. 
A

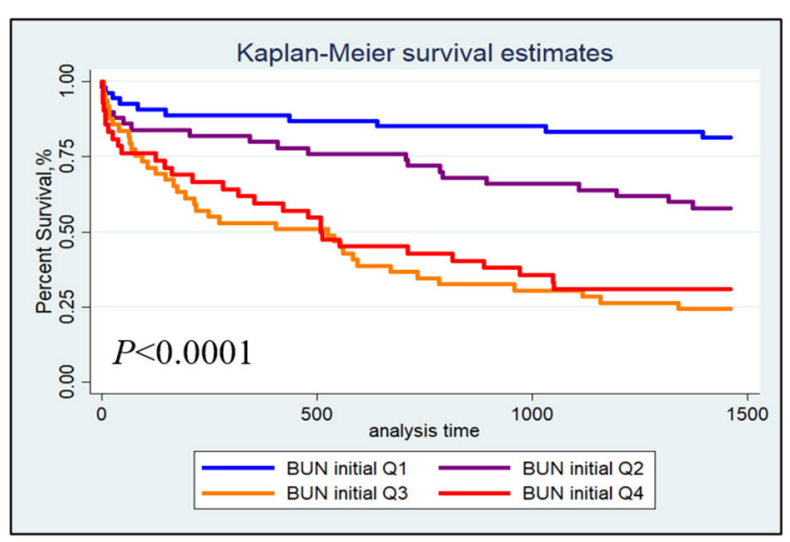

C

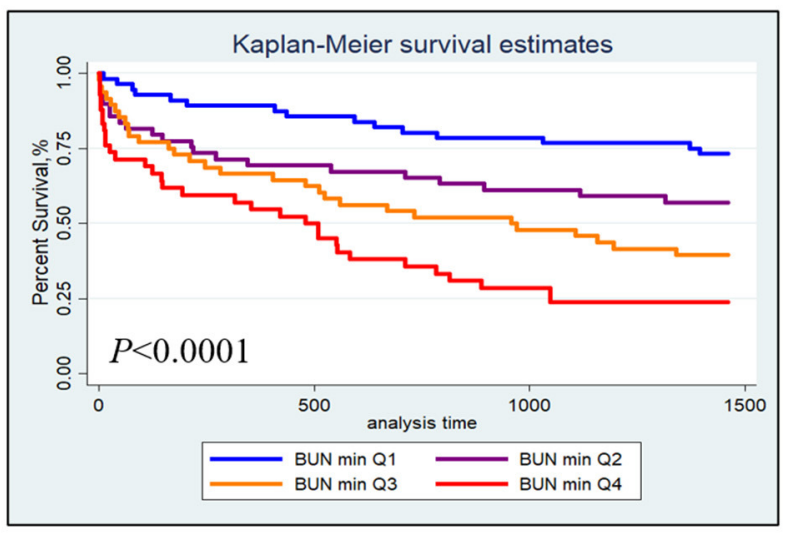

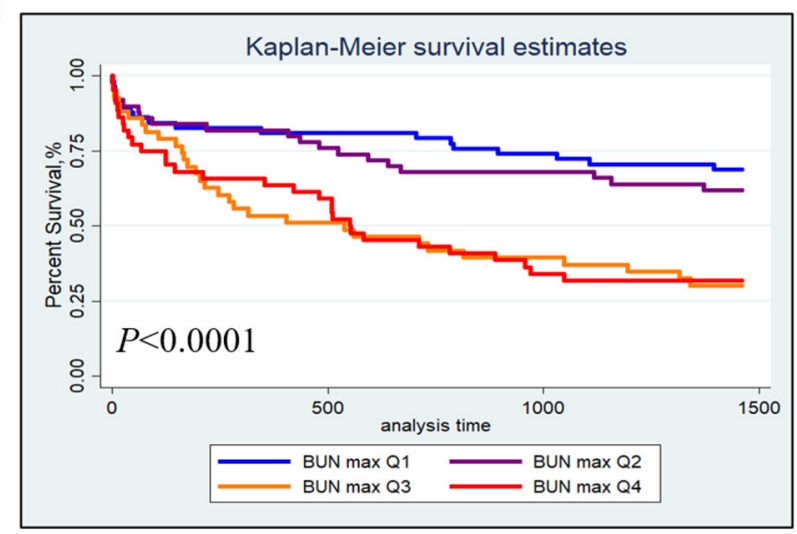

D

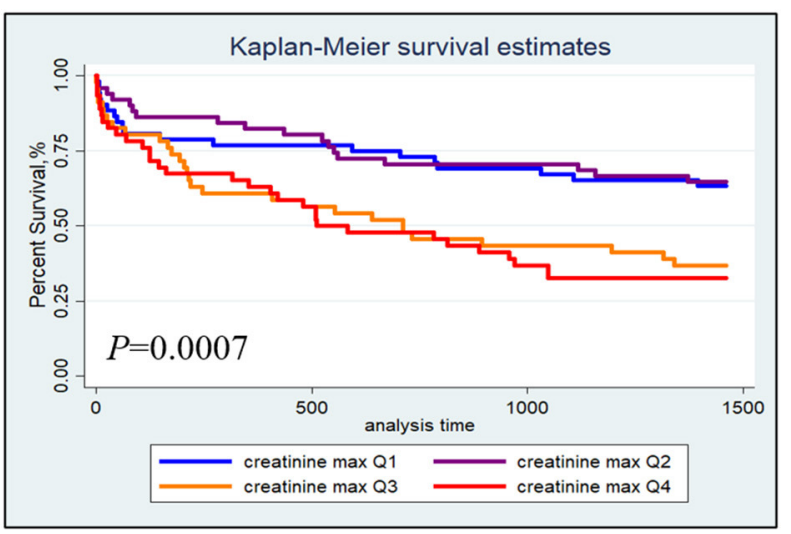

FIGURE 1 | Kaplan-Meier survival analysis plot for 4-year overall survival within different quartiles. (A) BUN initial; (B) BUN max; (C) BUN min; (D) creatinine max.

TABLE 6 | Univariate logistic regression analyses for prognosis in PPH patients.

\begin{tabular}{|c|c|c|c|c|c|c|}
\hline \multirow[b]{2}{*}{ Variable } & \multicolumn{2}{|c|}{ Hospital mortality } & \multicolumn{2}{|c|}{ 90-day mortality } & \multicolumn{2}{|c|}{ 4-year mortality } \\
\hline & OR (95\% Cl) & $P$ & OR $(95 \% \mathrm{Cl})$ & $P$ & OR (95\% Cl) & $P$ \\
\hline BUN initial, mg/dL & 1.01 (1.00-1.03) & 0.059 & $1.01(1.00-1.02)$ & 0.112 & 1.06 (1.03-1.09) & $<0.001$ \\
\hline BUN max, mg/dL & 1.02 (1.01-1.03) & 0.002 & $1.01(1.00-1.02)$ & 0.007 & $1.02(1.01-1.04)$ & $<0.001$ \\
\hline BUN min, mg/dL & 1.04 (1.02-1.06) & $<0.001$ & 1.02 (1.01-1.05) & 0.010 & 1.06 (1.03-1.09) & $<0.001$ \\
\hline Creatinine max, mg/dL & 1.30 (1.09-1.54) & 0.003 & $1.16(1.00-1.37)$ & 0.075 & 1.45 (1.12-1.87) & 0.005 \\
\hline Liver disease & 7.01 (2.86-17.15) & $<0.001$ & $4.57(1.94-10.74)$ & $<0.001$ & 2.35 (0.79-7.04) & 0.126 \\
\hline Renal failure & $0.70(0.26-1.90)$ & 0.480 & $0.40(0.15-1.08)$ & 0.070 & 2.08 (0.88-4.92) & 0.097 \\
\hline
\end{tabular}

For 4-year mortality, only patients in the CareVue system who were followed for at least 4-year were analyzed. The bold values represents a $P$ value $<0.05$.

The correlation of BUN with 90-day mortality in $\mathrm{PPH}$ patients was also investigated. As shown in Table 4, only BUN min and creatinine max were significantly associated with 90-day mortality (BUN min: $P=0.025$; creatinine max: $P=0.040$ ). Higher 90-day mortality was observed in the third and fourth BUN min quartiles of patients, while a higher rate was observed in the fourth creatinine max quartile of patients (Table 4).

For 4-year mortality, only patients in the CareVue system who were followed for at least 4 years were analyzed. The correlation of BUN and 4-year mortality is shown in Table 5. For initial BUN, higher 4-year mortality was observed in the third and the fourth
BUN min quartiles of patients, while the lowest 4-year mortality was observed in the first quartile of patients $(P<0.001)$. For BUN max, the 4-year mortality of the third and fourth quartiles was higher than that of the first and second quartiles $(P<0.001)$. For BUN min, a higher rate of 4-year mortality was observed in the higher quartile of patients $(P<0.001)$. Moreover, for creatinine max, the 4-year mortality of the third and fourth quartiles was higher than that of the first and second quartiles $(P=0.001)$

(Table 5).

The Kaplan-Meier survival curves comparing patients within different BUN and creatinine quartiles are shown in Figure $\mathbf{1 .}$ 
TABLE 7 | Association between BUN and creatinine with prognosis of PPH patients.

\begin{tabular}{|c|c|c|c|c|}
\hline \multicolumn{2}{|c|}{ Outcome } & \multirow[t]{2}{*}{ OR } & \multirow[t]{2}{*}{$95 \% \mathrm{Cl}$} & \multirow[t]{2}{*}{$\boldsymbol{P}$} \\
\hline \multicolumn{2}{|c|}{ Hospital mortality } & & & \\
\hline \multirow[t]{3}{*}{ BUN max } & Model 1 & 1.02 & $1.00-1.04$ & 0.013 \\
\hline & Model 2 & 1.02 & $1.00-1.03$ & 0.018 \\
\hline & Model 3 & 1.02 & $1.01-1.04$ & 0.012 \\
\hline \multirow[t]{3}{*}{ BUN min } & Model 1 & 1.05 & $1.02-1.08$ & 0.001 \\
\hline & Model 2 & 1.04 & $1.02-1.07$ & 0.001 \\
\hline & Model 3 & 1.05 & $1.02-1.08$ & 0.001 \\
\hline \multirow[t]{3}{*}{ Creatinine max } & Model 1 & 1.24 & $1.00-1.55$ & 0.061 \\
\hline & Model 2 & 1.17 & $0.96-1.43$ & 0.111 \\
\hline & Model 3 & 1.05 & $1.02-1.08$ & 0.001 \\
\hline \multicolumn{5}{|c|}{ 90-day mortality } \\
\hline \multirow[t]{3}{*}{ BUN max } & Model 1 & 1.01 & $1.00-1.02$ & 0.183 \\
\hline & Model 2 & 1.01 & $1.00-1.02$ & 0.064 \\
\hline & Model 3 & 1.01 & $1.00-1.02$ & 0.167 \\
\hline \multirow[t]{3}{*}{ BUN min } & Model 1 & 1.02 & $1.00-1.04$ & 0.074 \\
\hline & Model 2 & 1.02 & $1.00-1.05$ & 0.027 \\
\hline & Model 3 & 1.02 & $1.00-1.04$ & 0.077 \\
\hline \multirow[t]{3}{*}{ Creatinine max } & Model 1 & 1.01 & $0.82-1.24$ & 0.080 \\
\hline & Model 2 & 1.03 & $0.85-1.24$ & 0.758 \\
\hline & Model 3 & 1.01 & $0.83-1.25$ & 0.889 \\
\hline \multicolumn{5}{|l|}{ 4-year mortality } \\
\hline \multirow[t]{3}{*}{ BUN initial } & Model 1 & 1.02 & $1.00-1.03$ & 0.023 \\
\hline & Model 2 & 1.02 & $1.01-1.04$ & 0.003 \\
\hline & Model 3 & 1.02 & $1.00-1.03$ & 0.025 \\
\hline \multirow[t]{3}{*}{ BUN max } & Model 1 & 1.02 & $1.00-1.03$ & 0.014 \\
\hline & Model 2 & 1.02 & $1.01-1.04$ & 0.001 \\
\hline & Model 3 & 1.02 & $1.00-1.03$ & 0.011 \\
\hline \multirow[t]{3}{*}{ BUN min } & Model 1 & 1.05 & $1.02-1.08$ & 0.001 \\
\hline & Model 2 & 1.06 & $1.03-1.09$ & $<0.001$ \\
\hline & Model 3 & 1.05 & $1.02-1.08$ & 0.001 \\
\hline \multirow[t]{3}{*}{ Creatinine max } & Model 1 & 1.28 & $1.00-1.63$ & 0.053 \\
\hline & Model 2 & 1.39 & $1.08-1.78$ & 0.011 \\
\hline & Model 3 & 1.31 & $1.02-1.70$ & 0.036 \\
\hline
\end{tabular}

Model 1 was adjusted for $\mathrm{RR}, \mathrm{SpO}_{2}$, liver disease, valvular disease, SAPS II score and SBP. Model 2 was adjusted for RR, $\mathrm{SpO}_{2}$ and SOFA score. Model 3 was adjusted for $R R, \mathrm{SpO}_{2}$, liver disease, valvular disease, SAPS II score, SBP and SOFA score. For 4year mortality, only patients in the CareVue system who were followed for at least 4-year were analyzed. The bold values represents a $P$ value $<0.05$.

Figures 1A,B show that patients in the third and fourth quartiles BUN initial/max had the lowest survival (BUN initial: $P<0.0001$; BUN max: $P<0.0001)$. Figure $1 C$ shows that patients in the higher BUN min quartile tended to have lower 4-year survival ( $P$ $<0.0001)$. Figure 1D shows that patients in the third and fourth creatinine max quartiles had lower survival than those in the first and second creatinine max quartiles.

A univariate logistic regression analysis was performed. As shown in Table 6, initial BUN was associated with 4-year mortality $(\mathrm{OR}=1.06,95 \% \mathrm{CI}=1.03-1.09, P<0.001)$. BUN max was associated with hospital mortality $(\mathrm{OR}=1.02,95 \% \mathrm{CI}$ $=1.01-1.03, P=0.002)$, 90-day mortality $(\mathrm{OR}=1.01,95 \% \mathrm{CI}$
$=1.00-1.02, P=0.007)$ and 4 -year mortality $(\mathrm{OR}=1.02,95 \%$ $\mathrm{CI}=1.01-1.04, P<0.001)$. BUN min was also associated with hospital mortality $(\mathrm{OR}=1.04,95 \% \mathrm{CI}=1.02-1.06, P<0.001)$, 90-day mortality $(\mathrm{OR}=1.02,95 \% \mathrm{CI}=1.01-1.05, P=0.010)$ and 4 -year mortality $(\mathrm{OR}=1.06,95 \% \mathrm{CI}=1.03-1.09, P<0.001)$. Creatinine max was associated with hospital mortality $(\mathrm{OR}=$ $1.30,95 \% \mathrm{CI}=1.09-1.54, P=0.003)$ and 4 -year mortality $(\mathrm{OR}=$ $1.45,95 \% \mathrm{CI}=1.12-1.87, P=0.005)$. Liver disease was associated with hospital mortality $(\mathrm{OR}=7.01,95 \% \mathrm{CI}=2.86-17.15, P<$ $0.001)$ and 90 -day mortality $(\mathrm{OR}=4.57,95 \% \mathrm{CI}=1.94-10.74$, $P<0.001$ ), while no statistical association was observed between renal failure and mortality in $\mathrm{PPH}$ patients.

The results of the multivariate analyses are summarized in Table 7. In the multivariate analysis, Model 1 was adjusted for $\mathrm{RR}, \mathrm{SpO}_{2}$, liver disease, valvular disease, SAPS II score and SBP. Model 2 was adjusted for RR, $\mathrm{SpO}_{2}$ and SOFA score. Model 3 was adjusted for $\mathrm{RR}, \mathrm{SpO}_{2}$, liver disease, valvular disease, SAPS II score, SBP and SOFA score. BUN max (Model 1: OR $=1.02$, 95\% CI $=1.00-1.04, P=0.013$; Model $2: \mathrm{OR}=1.02,95 \% \mathrm{CI}=$ 1.00-1.03, $P=0.018$; Model 3: $\mathrm{OR}=1.02,95 \% \mathrm{CI}=1.01-1.04$, $P=0.012$ ) and BUN min (Model $1: \mathrm{OR}=1.05,95 \% \mathrm{CI}=1.02-$ 1.08, $P=0.001$; Model 2: $\mathrm{OR}=1.04,95 \% \mathrm{CI}=1.02-1.07, P=$ 0.001; Model 3: $\mathrm{OR}=1.05,95 \% \mathrm{CI}=1.02-1.08, P=0.001)$ were all positively associated with hospital mortality for all models. Moreover, only BUN min was associated with 90-day mortality for Model 2. Furthermore, BUN initial (Model 1: OR $=1.02,95 \%$ $\mathrm{CI}=1.00-1.03, P=0.023$; Model $2: \mathrm{OR}=1.02,95 \% \mathrm{CI}=1.01-$ 1.04, $P=0.003$; Model 3: $\mathrm{OR}=1.02,95 \% \mathrm{CI}=1.00-1.03, P=$ 0.025 ), BUN max (Model 1: OR $=1.02,95 \% \mathrm{CI}=1.00-1.03, P=$ 0.014; Model 2: $\mathrm{OR}=1.02,95 \% \mathrm{CI}=1.01-1.04, P=0.001$; Model 3: $\mathrm{OR}=1.02,95 \% \mathrm{CI}=1.00-1.03, P=0.011)$ and $\mathrm{BUN} \min$ (Model 1: OR $=1.05,95 \% \mathrm{CI}=1.02-1.08, P=0.001$; Model 2: $\mathrm{OR}=1.06,95 \% \mathrm{CI}=1.03-1.09, P<0.001 ;$ Model 3: OR = 1.05, $95 \% \mathrm{CI}=1.02-1.08, P=0.001)$ were positively associated with 4 year mortality in all models. Creatinine max was only associated with only 4-year mortality for Model 2 (Table 7).

\section{Predictive Ability of BUN for Prognosis of PPH}

The diagnostic value of BUN was examined using ROC curves. For hospital mortality, the diagnostic performance of BUN was moderately good (initial BUN AUC $=0.623$; maximum BUN $\mathrm{AUC}=0.656$; minimum BUN AUC $=0.694$ ) (Figure 2A). For 90-day mortality, the diagnostic performance of BUN was also moderately good (initial BUN AUC $=0.610$; maximum BUN $\mathrm{AUC}=0.615$; minimum BUN AUC $=0.644)$ (Figure 2B). For 4-year mortality, the diagnostic performance of BUN was better than that of hospital and 90-day mortalities (initial BUN AUC =0.756; maximum $\mathrm{BUN}$ AUC $=0.715$; minimum $\mathrm{BUN}$ AUC $=$ 0.736) (Figure 2C).

\section{The Prognostic Significance of the Ratio of BUN to Creatinine for PPH}

For deeper understanding the association of BUN and creatinine with prognosis of $\mathrm{PPH}$, the univariate and multivariate logistic regressions were used to analyze the ratio of BUN to 

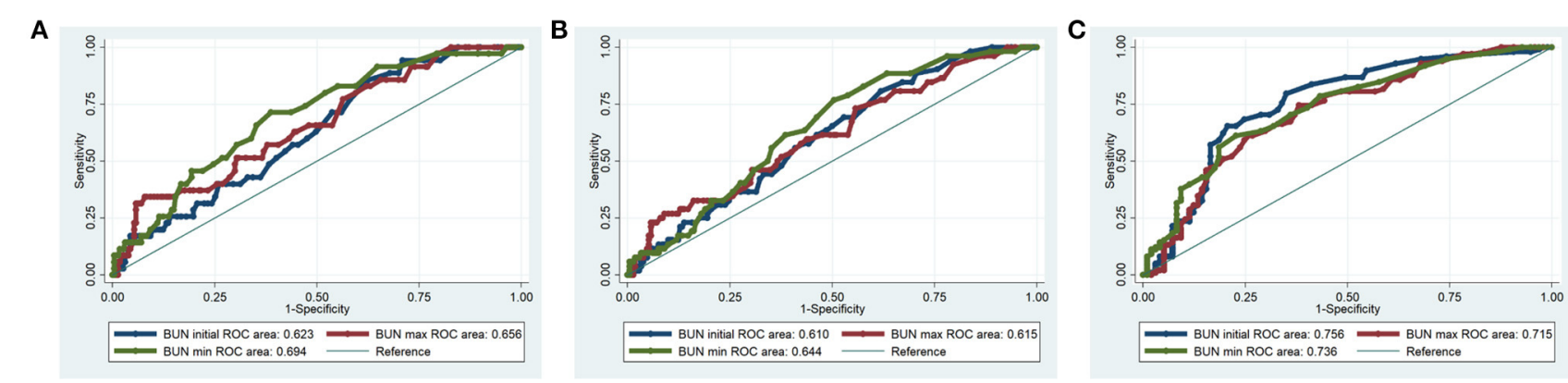

FIGURE 2 | ROC curves for BUN in hospital mortality, 90-day mortality and 4-year mortality of the PPH patients. (A) Hospital mortality; (B) 90-day mortality; (C) 4-year mortality.

creatinine. As shown in Table 8, only the ratio of BUN(min) to creatinine(min) was associated with hospital mortality (Univariate: $\mathrm{OR}=1.06,95 \% \mathrm{CI}=1.02-1.09, P=0.001$; Model 1: $\mathrm{OR}=1.05,95 \% \mathrm{CI}=1.01-1.09, P=0.014$; Model 2: $\mathrm{OR}=1.06$, 95\% CI $=1.02-1.11, P=0.001$; Model 3: OR $=1.05,95 \% \mathrm{CI}$ $=1.01-1.09, P=0.014)$. Moreover, the ratio of BUN(initial) to creatinine(initial) and the ratio of $\mathrm{BUN}(\mathrm{min})$ to creatinine(min) were positively associated with 90-day mortality [For the ratio of BUN(initial) to creatinine(initial), Univariate: $\mathrm{OR}=1.05,95 \%$ $\mathrm{CI}=1.02-1.08, P<0.001$; Model $1: \mathrm{OR}=1.04,95 \% \mathrm{CI}=$ $1.01-1.07, P=0.005$; Model 2 : $\mathrm{OR}=1.05$, 95\% CI $=1.02-$ 1.09, $P<0.001$; Model 3: $\mathrm{OR}=1.04,95 \% \mathrm{CI}=1.01-1.07, P=$ 0.006 . For the ratio of $\mathrm{BUN}(\mathrm{min})$ to creatinine $(\mathrm{min})$, Univariate: $\mathrm{OR}=1.07,95 \% \mathrm{CI}=1.04-1.10, P<0.001$; Model $1: \mathrm{OR}=$ $1.07,95 \% \mathrm{CI}=1.03-1.10, P<0.001$; Model 2 : OR $=1.08,95 \%$ $\mathrm{CI}=1.05-1.12, P<0.001$; Model 3: OR $=1.07,95 \% \mathrm{CI}=$ $1.03-1.11, P<0.001]$. Furthermore, the ratio of BUN(initial) to creatinine(initial) and the ratio of $\mathrm{BUN}(\mathrm{min})$ to creatinine(min) were positively associated with 4-year mortality [For the ratio of $\mathrm{BUN}$ (initial) to creatinine(initial), Univariate: OR $=1.06,95 \% \mathrm{CI}$ $=1.02-1.10, P=0.002$; Model $1: \mathrm{OR}=1.05,95 \% \mathrm{CI}=1.01-1.10$, $P=0.014$; Model 2: $\mathrm{OR}=1.06,95 \% \mathrm{CI}=1.02-1.10, P=0.002$; Model 3: $\mathrm{OR}=1.05,95 \% \mathrm{CI}=1.01-1.10, P=0.017$. For the ratio of BUN(min) to creatinine( $\mathrm{min})$, Univariate: $\mathrm{OR}=1.07,95 \% \mathrm{CI}$ $=1.03-1.12, P<0.001$; Model $1: \mathrm{OR}=1.07,95 \% \mathrm{CI}=1.03-1.13$, $P=0.002$; Model 2: $\mathrm{OR}=1.08,95 \% \mathrm{CI}=1.04-1.12, P<0.001$; Model 3: $\mathrm{OR}=1.07,95 \% \mathrm{CI}=1.03-1.13, P=0.003$ ] (Table 8).

\section{DISCUSSION}

$\mathrm{PAH}$ is a life-threatening disease associated with increased mortality regardless of the classification and underlying etiology $(13,14)$. Moreover, $\mathrm{PAH}$ is a progressive pulmonary circulatory disease characterized by vascular remodeling followed by increased pulmonary vascular resistance (PVR) and pulmonary artery pressure (PAP) that can lead to right ventricular (RV) overload and failure, ultimately resulting in premature death (15). The survival rate of PAH is between 68 and $93 \%$ at 1 year and 39 and $77 \%$ at 3 years $(13,16)$.

Many biomarkers have been investigated in $\mathrm{PH}$ (17), but only $\mathrm{BNP}$ and N-terminal pro-brain natriuretic peptide (NT-proBNP) have been widely used in routine practice and clinical trials, both of which are correlated with myocardial dysfunction, provide prognostic information at diagnosis and during follow-up, and have been incorporated into risk scores (18). A series novel serum markers were found to be the potential markers for PAH. Higher provirus integration site for Moloney murine leukemia virus (Pim-1) levels of PAH patients, and the correlations with NTproBNP, cardiac index and PVR and independently predicted mortality were observed (19). Serum IP-10 is elevated in the systemic sclerosis-associated $\mathrm{PAH}$, and there were correlations to clinical and hemodynamic measurements, and a link with survival was indicated (20). Red blood cell distribution width, although not specific to PAH, has been found to perform better than various other serum biomarkers at predicting survival. It also predicted survival independently of a six min walk distance and NT-proBNP (21). In the present study, it is proposed that BUN can be a novel potential prognostic predictor for PPH. BUN was positively correlated with the length of hospital stay and ICU stay of PPH patients. Higher BUN levels were associated with higher hospital mortality, 90-day mortality and 4-year mortality, as well as lower 4-year survival, of PPH patients. BUN could serve as an independent predictor of hospital, 90-day and 4-year mortality in PPH. To our knowledge, this is the first report about the association of BUN with the prognosis of $\mathrm{PPH}$.

BUN and creatinine were important detection molecules that indicated early renal injury and renal histological lesions (22). As far as we knew, there was currently little study linking creatinine and BUN to the prognosis of $\mathrm{PH}$. In our investigation, the ratio of BUN to creatinine was correlated with hospital, 90-day and 4-year mortalities for PPH (Table 8), suggesting their unknown involvement in $\mathrm{PPH}$. Kalpana S Mehta et al. showed that the prevalence of $\mathrm{PH}$ increased as chronic kidney disease stage advanced, and there was a positive correlation between $\mathrm{PH}$ and duration of chronic kidney disease, BUN and serum creatinine (23). Zhang et al. showed similar results, in which $\mathrm{PH}$ was significantly associated with end-stage renal disease, however, the level of BUN and creatinine were not associated with $\mathrm{PH}$ (24). Unlike their reports, renal failure was not associated with mortality in $\mathrm{PPH}$ patients in our study (Table 6), which might be because the secondary $\mathrm{PH}$ caused by renal injury has a completely different mechanism from the renal injury caused 
TABLE 8 | Association between the ratio of BUN to creatinine with prognosis of $\mathrm{PPH}$ patients.

\begin{tabular}{|c|c|c|c|c|}
\hline \multicolumn{2}{|l|}{ Outcome } & \multirow[t]{2}{*}{ OR } & \multirow[t]{2}{*}{$95 \% \mathrm{Cl}$} & \multirow[t]{2}{*}{$P$} \\
\hline \multicolumn{2}{|l|}{ Hospital mortality } & & & \\
\hline \multirow[t]{4}{*}{ The ratio of BUN(initial) to creatinine(initial) } & Univariate & 1.02 & $0.99-1.05$ & 0.113 \\
\hline & Model 1 & 1.01 & $0.98-1.05$ & 0.417 \\
\hline & Model 2 & 1.03 & $0.99-1.06$ & 0.111 \\
\hline & Model 3 & 1.01 & $0.98-1.05$ & 0.439 \\
\hline \multirow[t]{4}{*}{ The ratio of $\mathrm{BUN}(\max )$ to creatinine(max) } & Univariate & 0.98 & $0.95-1.02$ & 0.420 \\
\hline & Model 1 & 0.99 & $0.94-1.03$ & 0.486 \\
\hline & Model 2 & 0.99 & $0.96-1.03$ & 0.658 \\
\hline & Model 3 & 0.98 & $0.94-1.03$ & 0.465 \\
\hline \multirow[t]{4}{*}{ The ratio of $\mathrm{BUN}(\mathrm{min})$ to creatinine(min) } & Univariate & 1.06 & $1.02-1.09$ & 0.001 \\
\hline & Model 1 & 1.05 & $1.01-1.09$ & 0.014 \\
\hline & Model 2 & 1.06 & $1.02-1.11$ & 0.001 \\
\hline & Model 3 & 1.05 & $1.01-1.09$ & 0.014 \\
\hline \multicolumn{5}{|l|}{ 90-day mortality } \\
\hline \multirow[t]{4}{*}{ The ratio of BUN(initial) to creatinine(initial) } & Univariate & 1.05 & $1.02-1.08$ & $<0.001$ \\
\hline & Model 1 & 1.04 & $1.01-1.07$ & 0.005 \\
\hline & Model 2 & 1.05 & $1.02-1.09$ & $<0.001$ \\
\hline & Model 3 & 1.04 & $1.01-1.07$ & 0.006 \\
\hline \multirow[t]{4}{*}{ The ratio of $\mathrm{BUN}(\max )$ to creatinine(max) } & Univariate & 1.02 & $0.99-1.04$ & 0.127 \\
\hline & Model 1 & 1.02 & $0.99-1.05$ & 0.120 \\
\hline & Model 2 & 1.02 & $1.00-1.05$ & 0.043 \\
\hline & Model 3 & 1.02 & $0.99-1.05$ & 0.128 \\
\hline \multirow[t]{4}{*}{ The ratio of $\mathrm{BUN}(\mathrm{min})$ to creatinine(min) } & Univariate & 1.07 & $1.04-1.10$ & $<0.001$ \\
\hline & Model 1 & 1.07 & $1.03-1.10$ & $<0.001$ \\
\hline & Model 2 & 1.08 & $1.05-1.12$ & $<0.001$ \\
\hline & Model 3 & 1.07 & $1.03-1.11$ & $<0.001$ \\
\hline \multicolumn{5}{|l|}{ 4-year mortality } \\
\hline \multirow[t]{4}{*}{ The ratio of BUN(initial) to creatinine(initial) } & Univariate & 1.06 & $1.02-1.10$ & 0.002 \\
\hline & Model 1 & 1.05 & $1.01-1.10$ & 0.014 \\
\hline & Model 2 & 1.06 & $1.02-1.10$ & 0.002 \\
\hline & Model 3 & 1.05 & $1.01-1.10$ & 0.017 \\
\hline \multirow[t]{4}{*}{ The ratio of $\mathrm{BUN}(\max )$ to creatinine(max) } & Univariate & 1.03 & $1.00-1.05$ & 0.063 \\
\hline & Model 1 & 1.03 & $0.99-1.06$ & 0.110 \\
\hline & Model 2 & 1.03 & $1.00-1.06$ & 0.048 \\
\hline & Model 3 & 1.02 & $0.99-1.06$ & 0.140 \\
\hline \multirow[t]{4}{*}{ The ratio of $\mathrm{BUN}(\mathrm{min})$ to creatinine(min) } & Univariate & 1.07 & $1.03-1.12$ & $<0.001$ \\
\hline & Model 1 & 1.07 & $1.03-1.13$ & 0.002 \\
\hline & Model 2 & 1.08 & $1.04-1.12$ & $<0.001$ \\
\hline & Model 3 & 1.07 & $1.03-1.13$ & 0.003 \\
\hline
\end{tabular}

Model 1 was adjusted for RR, $\mathrm{SpO}_{2}$, liver disease, valvular disease, SAPS II score and SBP. Model 2 was adjusted for RR, SpO 2 and SOFA score. Model 3 was adjusted for $R R, \mathrm{SpO}_{2}$, liver disease, valvular disease, SAPS II score, SBP and SOFA score. For 4year mortality, only patients in the CareVue system who were followed for at least 4-year were analyzed. The bold values represents a $P$ value $<0.05$.

by $\mathrm{PPH}$. Although both BUN and creatinine were associated with the 4-year survival of PPH patients (Figure 1), only BUN could serve as an independent prognostic predictor of PPH in all models (Table 7), indicating that BUN might be more important than creatinine in $\mathrm{PPH}$, which was not only participated in renal injury, but also in other disease mechanisms in $\mathrm{PPH}$.
BUN in blood is the end product of nitrogen metabolism (25). The urea cycle pathway plays a major role in PAH severity and treatment response $(26,27)$. Urea and other elements of urea cycle, including AMP, 4-hydroxy-proline, ornithine and $\mathrm{N}$-acetylornithine, were demonstrated to be significantly upregulated in the PAH group (11). The main function of the urea cycle is to convert excess nitrogen in the form of ammonia to urea, which is excreted through the kidneys. L-arginine is an amino acid supplied by the urea cycle and is the link between the urea cycle and PAH. Low L-arginine levels could theoretically decrease nitric oxide synthesis and lead to $\mathrm{PH}$ (28). Moreover, previous studies demonstrated that polymorphisms in urea cycle enzyme genes were associated with PAH. The Thr1405 variant of carbamoyl-phosphate synthetase (CPS) was correlated with persistent pulmonary hypertension of the newborn (PPHN) (29). A single-nucleotide polymorphism (SNP) in arginase may be protective against pulmonary hypertension (30). Thus, the disorders of the urea cycle played a critical role in PAH; however, recent research has mainly focused on arginine or nitric oxide metabolites $(29,31)$ but not urea. Our data indicated that a higher urea level was correlated with poorer short-term and long-term survival rates. That might be because the urea level might reflect the degree of urea cycle disorder, which is directly related to the malignant progression of $\mathrm{PPH}$. According to the mention above, BUN can be a novel potential prognostic predictor for $\mathrm{PPH}$.

\section{CONCLUSION}

In summary, we demonstrated that BUN was positively correlated with the length of hospital stay and ICU stay of $\mathrm{PPH}$ patients. Higher BUN was associated with higher hospital mortality, 90-day mortality, and 4-year mortality, as well as lower 4-year survival of PPH patients. The diagnostic performance of BUN for mortality of PPH patient was moderately good. BUN could serve as an independent predictor of hospital, 90-day and 4-year mortality for PPH. Taken together, these results show that BUN can be a novel potential prognostic predictor for PPH.

\section{Limitation}

This was a single center, retrospective study with insufficient data. The disease definition of the MIMIC III database is based on the ICD-9-CM code. Therefore, some important information was lacking because of the limitations of the database itself, such as echocardiography results, electrocardiogram results, pulmonary artery pressure, risk scores, etc. The sample size of the study was small. More robust studies with larger sample size and metaanalyses should be done to further illustrate the relationship between BUN and PPH.

\section{DATA AVAILABILITY STATEMENT}

The datasets presented in this study can be found in online repositories. The names of the repository/repositories and accession number(s) can be found in the article/supplementary material. 


\section{ETHICS STATEMENT}

The institutional review boards of the MIT (Cambridge, Massachusetts) and BIDMC (Boston, Massachusetts) reviewed and approved studies involving human participants. According to national laws and institutional requirements, this study does not require written informed consent.

\section{AUTHOR CONTRIBUTIONS}

$\mathrm{JH}, \mathrm{BH}$, and GX conceived and designed the study. GX and $\mathrm{JH}$ administratively supported this work. GX, XinJ, DC, XQ, WL, LX, JZ, JT, and XiuJ provided, selected, assembled, analyzed, and interpreted data. All authors contributed toward data analysis, drafting and critically revising the paper, agree to be accountable

\section{REFERENCES}

1. Mandras SA, Mehta HS, Vaidya A. Pulmonary hypertension: a brief guide for clinicians. Mayo Clin Proc. (2020) 95:197888. doi: 10.1016/j.mayocp.2020.04.039

2. Kovacs G, Dumitrescu D, Barner A, Greiner S, Grunig E, Hager $A$, et al. Definition, clinical classification and initial diagnosis of pulmonary hypertension: updated recommendations from the Cologne Consensus Conference 2018. Int J Cardiol. (2018) 272S:11-9. doi: 10.1016/j.ijcard.2018.08.083

3. Simonneau G, Montani D, Celermajer DS, Denton CP, Gatzoulis MA, Krowka M, et al. Haemodynamic definitions and updated clinical classification of pulmonary hypertension. Eur Respir J. (2019) 53:1801913. doi: 10.1183/13993003.01913-2018

4. Girgis RE, Champion HC, Diette GB, Johns RA, Permutt S, Sylvester JT. Decreased exhaled nitric oxide in pulmonary arterial hypertension: response to bosentan therapy. Am J Respir Crit Care Med. (2005) 172:3527. doi: $10.1164 / \mathrm{rccm} .200412-1684 \mathrm{OC}$

5. Kaluarachchi DC, Smith CJ, Klein JM, Murray JC, Dagle JM, Ryckman KK. Polymorphisms in urea cycle enzyme genes are associated with persistent pulmonary hypertension of the newborn. Pediatr Res. (2018) 83:1427. doi: $10.1038 /$ pr.2017.143

6. Bruckdorfer R. The basics about nitric oxide. Mol Aspects Med. (2005) 26:331. doi: 10.1016/j.mam.2004.09.002

7. Morrell NW, Adnot S, Archer SL, Dupuis J, Lloyd Jones P, MacLean MR, et al. Cellular and molecular basis of pulmonary arterial hypertension. J Am Coll Cardiol. (2009) 54:S20-S31. doi: 10.1016/j.jacc.2009.04.018

8. Fulton DJR, Li X, Bordan Z, Haigh S, Bentley A, Chen F, et al. Reactive oxygen and nitrogen species in the development of pulmonary hypertension. Antioxidants. (2017) 6:54. doi: 10.3390/antiox6030054

9. Tabima DM, Frizzell S, Gladwin MT. Reactive oxygen and nitrogen species in pulmonary hypertension. Free Radic Biol Med. (2012) 52:197086. doi: 10.1016/j.freeradbiomed.2012.02.041

10. Maarsingh $\mathrm{H}$, Pera $\mathrm{T}$, Meurs $\mathrm{H}$. Arginase and pulmonary diseases. Naunyn Schmiedebergs Arch Pharmacol. (2008) 378:17184. doi: 10.1007/s00210-008-0286-7

11. Zheng HK, Zhao JH, Yan Y, Lian TY, Ye J, Wang XJ, et al. Metabolic reprogramming of the urea cycle pathway in experimental pulmonary arterial hypertension rats induced by monocrotaline. Respir Res. (2018) 19:94. doi: 10.1186/s12931-018-0800-5

12. Johnson AE, Pollard TJ, Shen L, Lehman LW, Feng M, Ghassemi M, et al. MIMIC-III, a freely accessible critical care database. Sci Data. (2016) 3:160035. doi: 10.1038/sdata.2016.35

13. Hoeper MM, Humbert M, Souza R, Idrees M, Kawut SM, Sliwa-Hahnle K, et al. A global view of pulmonary hypertension. Lancet Respir Med. (2016) 4:306-22. doi: 10.1016/S2213-2600(15)00543-3 for all aspects of the work, and have read and confirmed that they meet ICMJE criteria for authorship.

\section{FUNDING}

This work was financially supported by the National Natural Science Foundation of China (81900294 to JH) and the Major Transverse Research Projects of Jiaxing University, China (00619006 to GX).

\section{ACKNOWLEDGMENTS}

The authors greatly appreciate the editor and peer reviewers for their critical reading and insightful comments, which are helpful to improve our manuscript substantially.

14. Adler J, Gerhardt F, Wissmuller M, Adler C, Baldus S, Rosenkranz S. Pulmonary hypertension associated with left-sided heart failure. Curr Opin Cardiol. (2020) 35:610-9. doi: 10.1097/HCO.0000000000000791

15. Giuggioli D, Bruni C, Cacciapaglia F, Dardi F, De Cata A, Del Papa N, et al. Pulmonary arterial hypertension: guidelines and unmet clinical needs. Reumatismo. (2021) 72:228-46. doi: 10.4081/reumatismo.20 20.1310

16. Kim NH, Fisher M, Poch D, Zhao C, Shah M, Bartolome S. Long-term outcomes in pulmonary arterial hypertension by functional class: a metaanalysis of randomized controlled trials and observational registries. Pulm Circ. (2020) 10:2045894020935291. doi: 10.1177/2045894020 935291

17. McGlinchey N, Johnson MK. Novel serum biomarkers in pulmonary arterial hypertension. Biomark Med. (2014) 8:1001-11. doi: 10.2217/bmm.14.69

18. Lewis RA, Durrington C, Condliffe R, Kiely DG. BNP/NT-proBNP in pulmonary arterial hypertension: time for point-of-care testing? Eur Respir Rev. (2020) 29:200009. doi: 10.1183/16000617.0009-2020

19. Renard S, Paulin R, Breuils-Bonnet S, Simard S, Pibarot P, Bonnet S, et al. Pim1: A new biomarker in pulmonary arterial hypertension. Pulm Circ. (2013) 3:74-81. doi: 10.4103/2045-8932.109917

20. George PM, Oliver E, Dorfmuller P, Dubois OD, Reed DM, Kirkby NS, et al. Evidence for the involvement of type I interferon in pulmonary arterial hypertension. Circ Res. (2014) 114:677-88. doi: 10.1161/CIRCRESAHA.114.302221

21. Rhodes CJ, Wharton J, Howard LS, Gibbs JS, Wilkins MR. Red cell distribution width outperforms other potential circulating biomarkers in predicting survival in idiopathic pulmonary arterial hypertension. Heart. (2011) 97:1054-60. doi: 10.1136/hrt.2011.224857

22. van Meer L, Moerland M, Cohen AF, Burggraaf J. Urinary kidney biomarkers for early detection of nephrotoxicity in clinical drug development. Br J Clin Pharmacol. (2014) 77:947-57. doi: 10.1111/bcp.12282

23. Mehta KS, Shirkande AK, Bhurke SP, Pajai AE, Swami RS, Jadhav SN Pulmonary hypertension in various stages of chronic kidney disease in Indian patients. Indian J Nephrol. (2019) 29:95-101. doi: 10.4103/ijn.IJN_407_17

24. Zhang Y, Ding XH, Rao R, Wang Y, Pang F, Tang S, et al. The prevalence of pulmonary hypertension among maintenance dialysis patients with ESRD and its associated factors: a retrospective study. Front Med. (2020) 7:570874. doi: 10.3389/fmed.2020.570874

25. Aronson D, Mittleman MA, Burger AJ. Elevated blood urea nitrogen level as a predictor of mortality in patients admitted for decompensated heart failure. Am J Med. (2004) 116:466-73. doi: 10.1016/j.amjmed.2003.11.014

26. Chida A, Shintani M, Matsushita Y, Sato H, Eitoku T, Nakayama T, et al. Mutations of NOTCH3 in childhood pulmonary arterial hypertension. $\mathrm{Mol}$ Genet Genomic Med. (2014) 2:229-39. doi: 10.1002/mgg3.58

27. Dhaun N, Vachiery JL, Benza RL, Naeije R, Hwang LJ, Liu X, et al. Endothelin antagonism and uric acid levels in pulmonary arterial 
hypertension: clinical associations. J Heart Lung Transplant. (2014) 33:5217. doi: 10.1016/j.healun.2014.01.853

28. Xu W, Kaneko FT, Zheng S, Comhair SA, Janocha AJ, Goggans T, et al. Increased arginase II and decreased NO synthesis in endothelial cells of patients with pulmonary arterial hypertension. FASEB J. (2004) 18:17468. doi: 10.1096/fj.04-2317fje

29. Pearson DL, Dawling S, Walsh WF, Haines JL, Christman BW, Bazyk A, et al. Neonatal pulmonary hypertension-urea-cycle intermediates, nitric oxide production, and carbamoyl-phosphate synthetase function. $N$ Engl J Med. (2001) 344:1832-8. doi: 10.1056/NEJM2001061434 42404

30. Trittmann JK, Nelin LD, Zmuda EJ, Gastier-Foster JM, Chen B, Backes CH, et al. Arginase I gene single-nucleotide polymorphism is associated with decreased risk of pulmonary hypertension in bronchopulmonary dysplasia. Acta Paediatr. (2014) 103:e439-43. doi: 10.1111/apa.12717

31. Vosatka RJ, Kashyap S, Trifiletti RR. Arginine deficiency accompanies persistent pulmonary hypertension of the newborn. Biol Neonate. (1994) 66:65-70. doi: 10.1159/000244091
Conflict of Interest: The authors declare that the research was conducted in the absence of any commercial or financial relationships that could be construed as a potential conflict of interest.

Publisher's Note: All claims expressed in this article are solely those of the authors and do not necessarily represent those of their affiliated organizations, or those of the publisher, the editors and the reviewers. Any product that may be evaluated in this article, or claim that may be made by its manufacturer, is not guaranteed or endorsed by the publisher.

Copyright $\odot 2021 \mathrm{Hu}, \mathrm{Xu}$, Jin, Chen, Qian, Li, Xu, Zhu, Tang, Jin and Hou. This is an open-access article distributed under the terms of the Creative Commons Attribution License (CC BY). The use, distribution or reproduction in other forums is permitted, provided the original author(s) and the copyright owner(s) are credited and that the original publication in this journal is cited, in accordance with accepted academic practice. No use, distribution or reproduction is permitted which does not comply with these terms. 